\title{
False memories of emotional and neutral words
}

\author{
Jennifer El Sharkawy ${ }^{\mathrm{a}, 1,2}$, Katarina Groth ${ }^{\mathrm{a}, 1,3}$, Céline Vetter ${ }^{\mathrm{a}, 1,4}$, Anna Beraldi ${ }^{\mathrm{a}, \mathrm{b}}$ and Kristina Fast ${ }^{\mathrm{a}, \mathrm{b}, *}$ \\ ${ }^{a}$ Neuro-Cognitive Psychology, Department Psychology, Ludwig-Maximilians-Universit ät München, Germany \\ ${ }^{\mathrm{b}}$ Division of Clinical Psychology and Psychophysiology, Department of Psychiatry, \\ Ludwig-Maximilians-Universität München, Germany
}

\begin{abstract}
This study used the Deese-Roediger-McDermott paradigm [34] to investigate the direction and the extent to which emotional valence in semantic word lists influences the formation of false memories (FM). The experimental paradigm consisted of 1) a study phase (learning of neutral and negative lists of words semantically associated to a non-presented critical lure (CL), 2) a free recall phase, and 3) a recognition phase. Participants had to indicate whether the displayed item was "new" (new item or non-studied CL) or "old" (studied list item). CL associated with negative word lists elicited significantly more FM than CL associated with neutral word lists. This finding is in contrast to previous work showing that emotional words elicit fewer FM than neutral words. The results of our study also suggest that valence is capable of influencing emotional memory in terms of encoding and retrieval processes.
\end{abstract}

Keywords: False memory, emotion, valence, Deese-Roediger-McDermott paradigm

\section{Introduction}

It has been well established that memory for emotional events is different than for neutral events [2, 12,15-17,24]. These studies could all show that distinct neurobiological regions underlie memory of neutral and emotional content ${ }^{5}$. There is emerging ev-

\footnotetext{
${ }^{1}$ Contributed equally and are jointly considered first author.

${ }^{2}$ Current address: University Clinic for Children and Adolescent Psychiatry, Psychotherapy and Psychosomatic, LudwigMaximilians-University, Munich, Germany.

${ }^{3}$ Current address: Transfer Centre for Neuroscience and Learning, Institute for Neurosciences and Learning, University Hospital Ulm, Germany.

${ }^{4}$ Current address: Psychology Department of the University of Warwick, UK. Institute of Medical Psychology, LudwigMaximilians-University, Munich, Germany.

* Corresponding author: Dr. Kristina Fast, Department of Psychiatry, Division of Clinical Psychology and Psychophysiology, Section of Neurocognition, Ludwig-Maximilians-University, Munich, Nußbaumstr. 7, D- 80336 München. Tel.: +49 895160 5565; Fax: +49 895160 5562; E-mail: kristina.fast@med.uni-muenchen.de.

${ }^{5}$ The term emotional content refers to stimuli that have been rated on a scale of arousal ("calm - exciting"), or on a scale of emotional valence ("pleasant - unpleasant") or a combination of both.
}

idence that not only neutral and emotional memory can be differentiated, but also that positive and negative content are subjected to distinct processing mechanisms. The valence hypothesis $[7,8]$ posits distinct neural underpinnings for the processing of emotional valent and neutral stimuli which is supported by recent neuroimaging studies (e.g. [13]). It is generally assumed that valence is a valid criterion to study memory processes [23,25].

The Deese-Roediger-McDermott (DRM) paradigm $[9,10,34]$ is widely used to study the phenomenon of false memories. Participants are typically presented with word lists highly associated to a non-presented word, the critical lure (CL). In subsequent recognition phases the CL is often mis-categorized as a presented word. Such events are referred to as false memories (FM). The DRM paradigm has been used to examine memory systems, including memory of language representations $[4,5,40]$, memory effects of aging $[11,14$, 22] and memory in clinical populations [27].

Pesta and colleagues [32] investigated whether CL derived from emotional content would be more distinct than neutral CL, and therefore less likely to be 


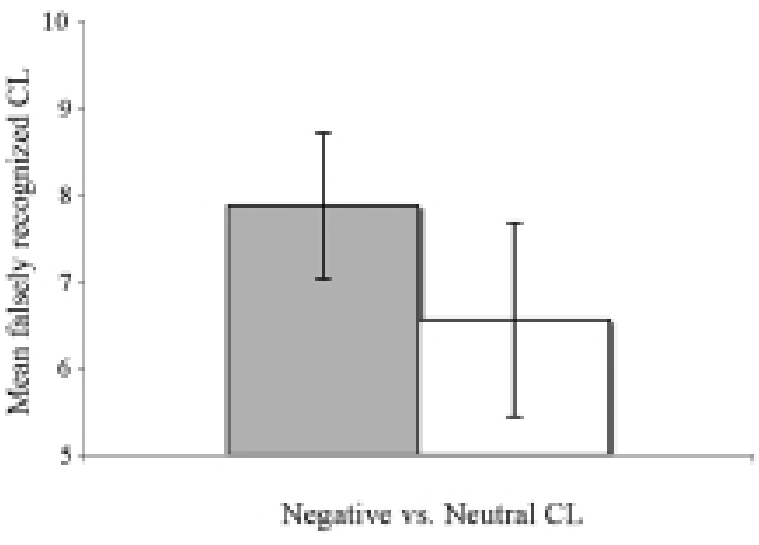

Fig. 1. This graph visualizes the mean number of falsely recognized words for negative and neutral non-presented associates (CL) during recognition phase. Subjects produced significantly more false alarms when the non-presented associate was a negative word.

falsely recognized. They found enhanced memory for emotional CL that were phonologically associated to lists of neutral, orthographically similar words in comparison to neutral CL. Importantly, the authors employed not only emotionally laden stimuli, but also taboo words (e.g. whore), which leads to enhanced recognition/rejection capabilities [38]. Thus, differential retrieval heuristics may be employed that would reduce false recall/recognition $[19,36]$.

The present study aims to investigate how semantically associated words, either neutral or of negative emotional valence, modulate the production of FM. Adolphs and Damasio [1] postulated that emotional situations were paramount to survival. Therefore, semantically relevant emotional content could create stronger inter-item associations. In turn, this would result in enhanced FM production, a prediction consistent with the Activation Monitoring Framework (a review is beyond the scope of this article, but see $[33,35])$.

\section{Method}

\subsection{Participants}

32 healthy volunteers ( 16 female) with a mean age of 26.2 years $(\mathrm{sd}=3.3 \mathrm{yrs})$ participated in the study. All were native German speakers with university education. Mean verbal IQ, assessed with the MWT-B [26], was $116.25(\mathrm{sd}=11.94)$.

\subsection{Material}

28 lists of converging associates were used, each consisting of 12 nouns (to avoid interference due to distinct neural processing of different word categories [6,31, 37]) strongly associated to a CL. All word lists were taken from the "Uni-Leipzig-database" [39]. In the case that insufficient nouns were supplied by the database $(<5 \%)$, backward association lists were created via an independent sample $(n=18)$. Previously, this has been shown to be a valid approach [3]. To sacrifice the semantic associative strength within the word lists, we included highly associated words despite differential word length, word frequency or distinct levels of word concreteness. These factors will be considered in the results section. Word concreteness was evaluated by a further independent sample $(n=12)$ who rated all words on a Likert scale from one to five $(1=$ highly concrete, 5 = highly abstract).

\subsection{Procedure}

The experiment consisted of a study phase, a free recall phase and a recognition phase (all proceeding in a subsequent manner) followed by the assessment of verbal IQ. During the study phase, all 28 negative and neutral word lists were presented on a computer screen for $15 \mathrm{sec}$ in a pseudo-randomized order. Sets were counter-balanced across participants. CL were not included in this phase. Participants were instructed to learn all words for a subsequent memory test. In the recall phase participants were asked to verbally list the remembered items (max. $5 \mathrm{~min}$ ) and experimenters recorded their responses. In the recognition phase, 60 words (20 old list items, $20 \mathrm{CL}$ and 20 unrelated words, each $50 \%$ neutral, $50 \%$ negative and pseudo-randomly selected) were successively presented (until response or max. $5 \mathrm{sec}$ ) via monitor and participants indicated whether the word was "old" (studied list item) or "new" (new unrelated word or CL). Responses were instructed to be given as spontaneously and as accurately as possible via mouse button press. The experiment lasted approximately $30 \mathrm{~min}$. Each participant completed all 3 phases.

\section{Results}

Two tailed t-tests for paired samples (alpha level $0.05)$ were calculated for free recall, recognition performance, and FM occurrence. The dependent variable 
Table 1

Descriptive Statistics: Word-Recognition

\begin{tabular}{lrrrrrrr}
\hline & \multicolumn{3}{c}{ Negative } & & \multicolumn{3}{c}{ Neutral } \\
\cline { 2 - 4 } \cline { 6 - 8 } & Old & CL & Intrusion & & Old & CL & Intrusion \\
\hline Mean & 5.47 & 7.88 & 1.34 & & 5.31 & 6.56 & 0.97 \\
SD & 1.92 & 1.68 & 1.33 & & 1.15 & 2.24 & 1.15 \\
correct & & 16.25 & & & & 17.84 & \\
\hline
\end{tabular}

Note: Old $=$ previously learned list item, $\mathrm{CL}=$ associated nonpresented item, Intrusion $=$ non-associated new item, $\mathrm{SD}=$ standard deviation.

was "response type" (correct $=$ correctly recognized old or new list item; incorrect $=$ false memory [CL], intrusions [new items] or failed recognition [old items]), the independent variable was "list type" (neutral versus negative CL-list).

\subsection{Recall}

Participants correctly remembered an average of 49.28 words. A comparison between the mean number of negative (mean $=21.31$, sd $=8.15$ ) and neutral words (mean $=22.75$, sd $=10.23$ ) recalled was non-significant, $[t(31)=-0.706, p=0.486]$. The mean number of negative and neutral CL in free recall (means $=1.81$ and 2.16 , sd $=1.35$ and 1.9 , respectively) did not differ significantly, $[t(31)=1.046$, $p=0.304]$. Participants were equally prone to intrusions, independent of negative $($ mean $=0.9, \mathrm{sd}=1.16)$ or neutral valence $($ mean $=0.77, \mathrm{sd}=1),[t(31)=$ $0.436, p=0.666]$.

\subsection{Recognition}

The descriptive statistics for recognition are depicted in Table 1. Mean number of correct responses $[t(31)=$ $0.524, p=0.604]$ and the mean number of intrusions $[t(31)=1.506, p=0.142]$ did not differ significantly between neutral and negative words. Negative CL were significantly more often falsely recognized than neutral $\mathrm{CL}[t(31)=2.860, p<0.01]$. Finally, the mean total correct recognition was significantly better for neutral words $[t(31)=-2.597, p<0.05]$. Cohen's d' statistic for dependent samples was calculated to evaluate the effect size $\left(d^{\prime}=0.66\right)$. Emotional valence thus appears to have a relatively large effect on false memory production. Figure 1 visualizes this main result.

The proportion of falsely recognized CL outnumbered the correct recognition of list items in the negative $[t(31)=-5.445, p<0.001]$ and neutral $[t(31)=$ $-2.968, p<0.01]$ conditions. To exclude the possibility that subjects performed at chance level and to assure that the lists had been learned, mean list item recognition was compared to the mean number of intrusions. The proportion of neutral list items recognized was significantly higher than for neutral intrusions $[t(31)=$ $17.778, p<0.001]$. The same was observed for negative list items $[t(31)=12.416, p<0.001]$ when compared to the number of negative intrusions.

Additionally, the factors word length, word frequency and level of concreteness were analysed. Word length of negative (mean $=1.79$ syllables, $\mathrm{sd}=0.96$ ) and neutral CL (mean $=1.5$, sd $=0.65)$ was comparable $[t(13)=1.000, p=0.336]$. However, negative list items $($ mean $=2.42$ syllables, $\mathrm{sd}=0.84$ ) were significantly longer than neutral ones (mean $=1.94$, sd $=1.03),[t(177)=4.801, p<0.000]$. Similarly, word concreteness for negative $\mathrm{CL}($ mean $=2.30, \mathrm{sd}=$ $0.78)$ and negative list items $($ mean $=2.27, \mathrm{sd}=0.91)$ was rated more abstract than for neutral CL (mean = 1.37 , sd $=0.40),[t(13)=4.904, p<0.000]$ and neutral list items $($ mean $=1.48, \mathrm{sd}=0.53),[t(177)=$ 10.774, $p<0.000$ ]. Word frequency of negative $($ mean $=17865.21, \mathrm{sd}=22765.29)$ and neutral CL $($ mean $=29937.93, \mathrm{sd}=61857.37)$ as well as of negative (mean $=16334.77$, $\mathrm{sd}=27919.92)$ and neutral list items ( mean $=21452.85$, $\mathrm{sd}=55965.46)$ was comparable $[t(13)=0.661, p=0.520 ; t(177)=1.113$, $p=0.267$; respectively].

\section{Discussion}

The main goal of this study was to compare the FM rates elicited from lists of words semantically associated with a negative or a neutral CL. We found that negative CL were more often falsely recognized, which is in contrast to previous work done in this field [20, 21,23,29,32] but in line with work done by Adolphs and Damasio [1] as well as theories like the Activation Monitoring Framework [33,35]. The results revealed that both negative and neutral CL were recognized more often than negative and neutral list items (a common finding, e.g. [30]) and that the correct recognition of list items was greater for old than for new items. These findings show that the word lists were successful in eliciting FM and that subjects did learn the lists.

With regard to the influence of word length in FM occurrence, negative words were significantly longer in this experiment than neutral items. Word length is commonly thought to increase word distinctiveness [35] and thus, one would expect lower frequency of FM. However, our results followed an opposite pattern. One 
might conclude that word length is less important in influencing FM occurrence than semantic associative strength and emotional valence.

Similarly, negative items were judged as being more abstract than neutral items. Previous studies [18,28] report higher false recognition rates with increasing levels of abstractness. This is in line with our results. It remains unclear whether negative words trigger more FM due to higher levels of abstractness or due to their emotional value. Therefore, this abstractness question deserves further attention in context with the DRM paradigm.

In conclusion, our results highlight a novel twist on a widely used memory paradigm. Additionally, the results are theoretically supported by the activation monitoring framework in that emotional content of words might also increase inter-item associations and thus decrease memory precision. Finally, our results raise numerous possibilities for future research endeavours.

\section{References}

[1] R. Adolphs and A.R. Damasio, Neurobiology of emotion at a systems level, in: The Neuropsychology of emotion, J.C. Borod, ed., Oxford University Press, Oxford, 2000, pp. 194213.

[2] R. Adolphs, D. Tranel and N. Denburg, Impaired emotional declarative memory following unilateral amygdala damage, Learning and Memory 7 (2000), 180-186.

[3] D. Anaki, Y. Faran, D. Ben Shalom and A. Henik, The false memory and the mirror effects: The role of familiarity and backward association in creating false recollections, Journal of Memory and Language 52 (2005), 87-102.

[4] L. Buchanan, N.R. Brown, R. Cabeza and C. Maitson, False memories and semantic lexicon arrangement, Brain and Language 68 (1999), 172-177.

[5] R. Cabeza and E.R. Lennartson, False memory across languages: Implicit associative response vs. fuzzy trace view, Memory 13 (2005), 1-5.

[6] A.R. Damasio and D. Tranel, Nouns and verbs are retrieved with differently distributed neural systems, Proceedings of the National Academy of Sciences of the United States of America 90 (1993), 4957-4960.

[7] R.J. Davidson, Cerebral asymmetry, emotion and affective style, in: Brain Asymmetry, R.J. Davidson and K. Hugdahl, eds, MIT Press, Cambridge, MA, 1995 pp. 361-387.

[8] R.J. Davidson and W. Irwin, The functional neuroanatomy of emotion and affective style, Trends in Cognitive Sciences $\mathbf{3}$ (1999), 11-20.

[9] J. Deese, Influence of inter-item associative strength upon immediate free recall, Psychological Reports 5 (1959), 305312.

[10] J. Deese, On the prediction of occurrence of particular verbal intrusions in immediate recall, Journal of Experimental Psychology 58 (1959), 17-22.

[11] S.A. Dewhurst and C.A. Robinson, False memories in children: Evidence for a shift from phonological to semantic associations, Psychological Science 15 (2004), 782-786.
[12] F. Dolcos and R. Cabeza, Event-related potentials of emotional memory: Encoding pleasant, unpleasant and neutral pictures, Cognitive, Affective, \& Behavioral Neuroscience 2 (2002), 252-263.

[13] F. Dolcos, K.S. LaBar and R. Cabeza, Dissociable effects of arousal and valence on prefrontal activity indexing emotional evaluation and subsequent memory: an event related fMRI study, Neuroimage 23 (2004), 64-74.

[14] D.A. Gallo and H.L. Roediger III., The effects of associations and aging on illusory recollection, Memory and Cognition 31 (2003), 1036-1044.

[15] S. Hamann, Cognitive and neural mechanisms of emotional memory, Trends in Cognitive Sciences 5 (2001), 394-400.

[16] S.B.Hamann, T.D. Ely, S.T. Grafton and C.D. Kilts, Amygdala activity related to enhanced memory for pleasant and aversive stimuli, Nature Neuroscience 2 (1999), 289-293.

[17] S.B. Hamann, E.S. Monarch and F.C. Goldstein, Memory enhancement for emotional stimuli is impaired in early Alzheimer's Disease, Neuropsychology 14 (2000), 82-92.

[18] E. Hirshman and J. Arndt, Discrimintating alternative conceptions of false recognition: The cases of word concertreeks and word frequency, Journal of Experimental Psychology: Memory, Learning \& Cognition 23 (1997), 1306-1323.

[19] L. Israel and D.L. Schacter, Pictorial encoding reduces false recognition of semantic associates, Psychonomic Bulletin \& Review 4 (1997), 577-581.

[20] E.A. Kensinger, Investigations of the cognitive and neural processes supporting memory for neutral and emotional words, Dissertation Abstracts International: Section B: The Sciences and Engineering 64 (2003).

[21] E.A. Kensinger and S. Corkin, Memory enhancement for emotional words: Are emotional words more vividly remembered than neutral words? Memory and Cognition 31 (2003), 1169 1180 .

[22] E.A. Kensinger and S. Corkin, The effects of emotional content and aging on false memories, Cognitive, Affective and Behavioral Neuroscience 4 (2004), 1-9.

[23] E.A. Kensinger and S. Corkin, Two routes to emotional memory: distinct neural processes for valence and arousal, Proceedings of the National Academy of Sciences of the United States of America 101 (2004), 3310-3315.

[24] E.A. Kensinger and D.L. Schacter, Amygdala activity is associated with the successful encoding of item, but not source information for positive and negative stimuli, Journal of Neuroscience 26 (2006), 2564-2570.

[25] K.S. LaBar and R. Cabeza, Cognitive neuroscience of emotional memory, Nature Reviews, Neuroscience 7 (2006), 5463.

[26] S. Lehrl, Mehrfach-Wortschatz-Intelligenztest, Version B, 5th Edition, Spitta Verlag, Balingen, 2005.

[27] S. Moritz, T.S. Woodward, C. Cuttler, J.C. Whitman and J.M. Watson, False memories in schizophrenia, Neuropsychology 18 (2004), 276-283.

[28] M. Nieves Perez-Mata, J. Don Read and M. Diges, Effects of divided attention and word concreteness on correct recall and false memory reports, Memory 10 (2002), 161-177.

[29] K.N. Ochsner, Are affective events richly recollected or simply familiar? The experience and process of recognizing feelings past, Journal of Experimental Psychology: General 129 (2000), 242-261.

[30] D.G. Payne, C.J. Elie, J.M. Blackwell and J.S. Neuschatz, Memory illusions: Recalling, recognizing, and recollecting events that never occurred, Journal of Memory \& Language 35 (1996), 261-285. 
[31] D. Perani, S.F. Cappa, T. Schnur, M. Tettamanti, S. Collina, M.M. Rosa and F. Fazio, The neural correlates of verb and noun processing: A PET study, Brain 122 (1999), 2337-2344.

[32] B.J. Pesta, M.D. Murphy and R.E. Sanders, Are emotionally charged lures immune to false memory? Journal of Experimental Psychology: Learning, Memory, and Cognition 27 (2001), 328-338.

[33] H.L. Roediger III., D.A. Balota and J.M. Watson, Spreading activation and the arousal of false memories, in: The nature of remembering: Essays in honour of Robert, G. Crowder, H.L. Roediger III., J.S. Naime, L. Neath and A.M. Surprenant, eds, American Psychological Association, Washington DC, 2005, pp. 95-115.

[34] H.L. Roediger III. and K.B. McDermott, Creating false memories: Remember words not presented in lists, Journal of Experimental Psychology: Learning, Memory, \& Cognition 21 (1995), 803-814.

[35] H.L. Roediger III., K.B. McDermott, J.M. Watson and A.D. Gallo, Factors that determine false recall: A multiple regression analysis, Psychonomic Bulletin and Review 8 (2001), $385-407$.
[36] D.L. Schacter, L. Israel and C. Racine, Suppressing false recognition in younger and older adults: the distinctiveness heuristics, Journal of Memory \& Language 40 (1999), 1-24.

[37] K.A. Shapiro, L.R. Moo and A. Caramazza, Cortical signatures of noun and verb production, Proceedings of the National Academy of Sciences of the United States of America 103 (2006), 1644-1649.

[38] J. Starns, J. Hicks and R. Marsh, Repetition effects in associative false recognition: Theme-based criterion shifts are the exception, not the rule, Memory 14 (2006), 742-761.

[39] University of Leipzig, Wortschatz Uni-Leipzig, http:// wortschatz.uni-leipzig.de Announcement posted on the World Wide Web, 2006.

[40] J.M. Watson, D.A. Balota and H.L. Roediger III, Creating false memories with hybrid lists of semantic and phonological associates: Over-additive false memories produced by converging associative networks, Journal of Memory and Language 49 (2003), 95-118. 


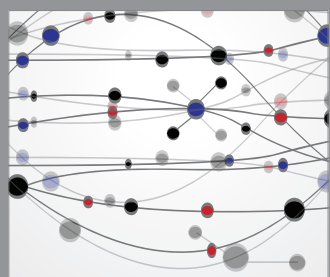

The Scientific World Journal
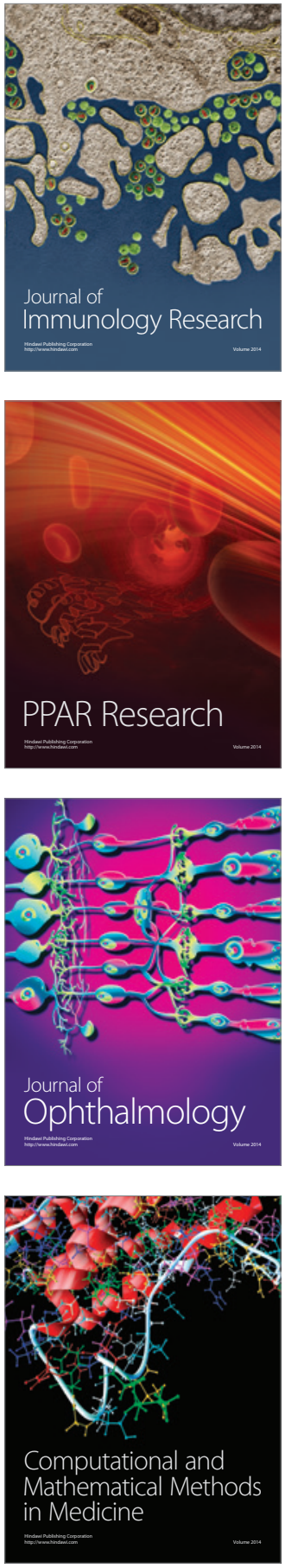

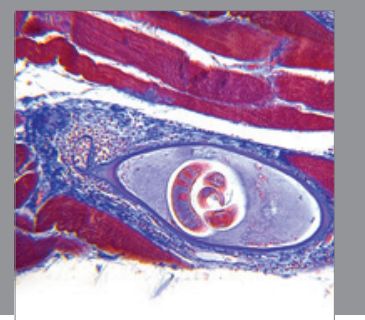

Gastroenterology

Research and Practice
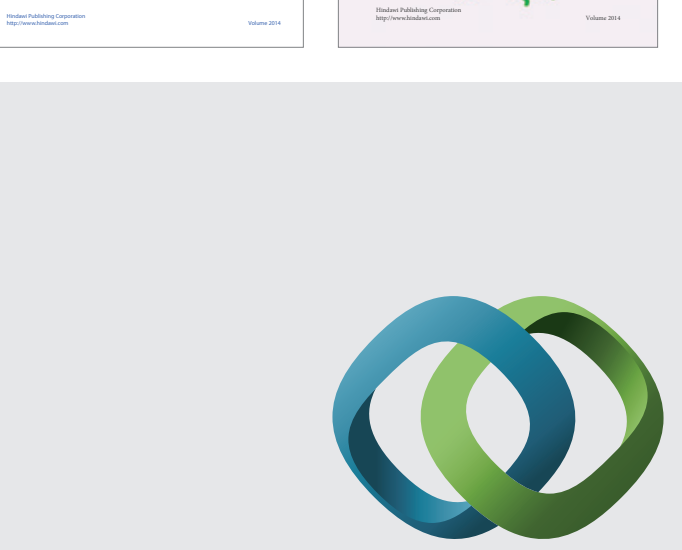

\section{Hindawi}

Submit your manuscripts at

http://www.hindawi.com
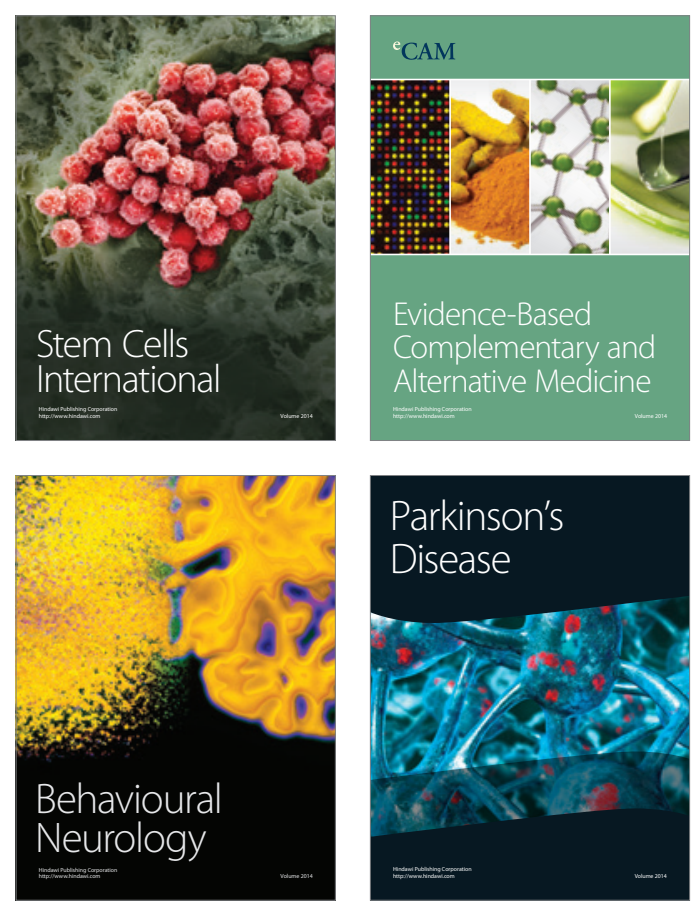

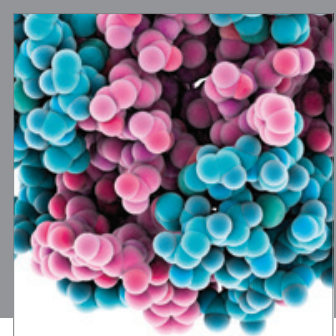

Journal of
Diabetes Research

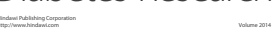

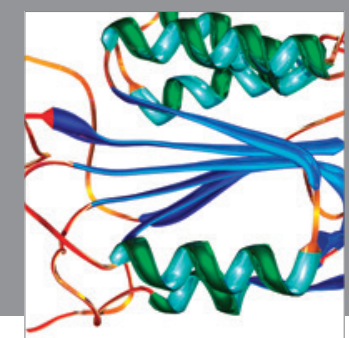

Disease Markers
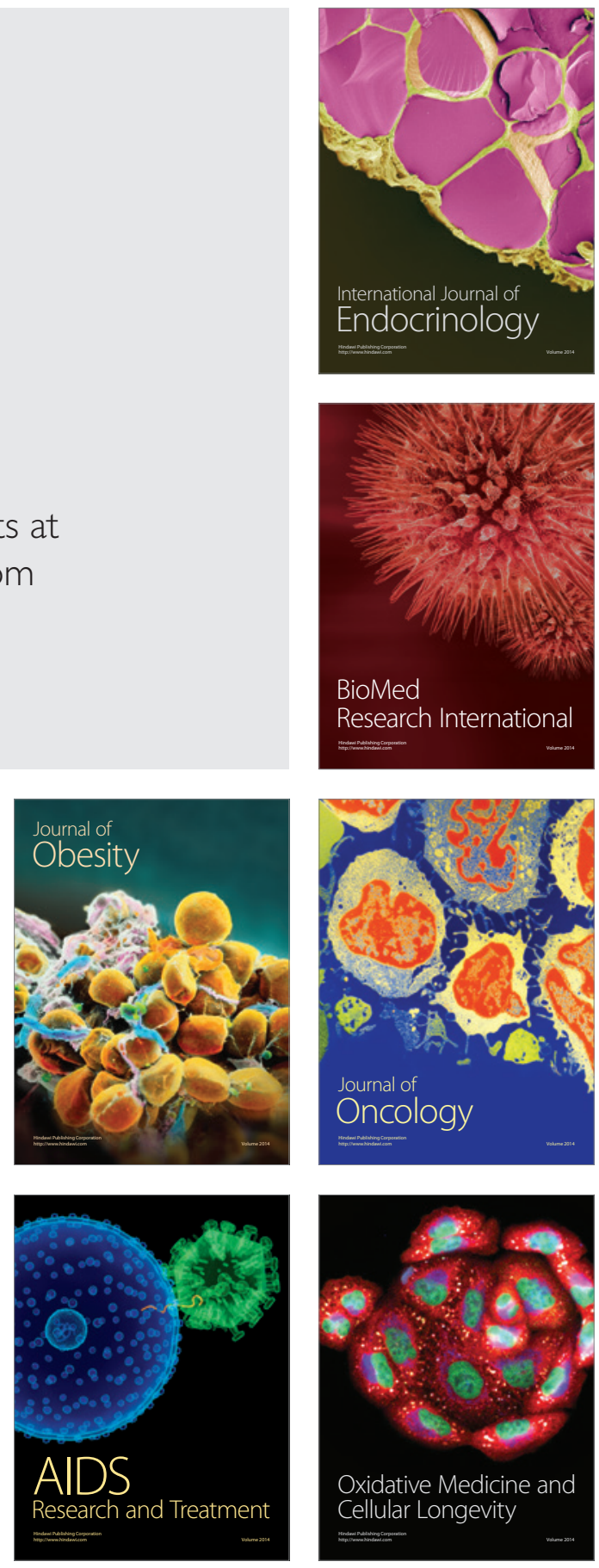